\title{
Wave Packet Description of Neutrino Oscillation in a Progenitor Star Supernova Environment
}

\author{
F. R. Torres and M. M. Guzzo \\ Instituto de Física "Gleb Wataghin", Universidade Estadual de Campinas, Unicamp, 13083-970, São Paulo, Brazil
}

Received on 13 September, 2007

\begin{abstract}
In this work we briefly review the wave packet approach of two generation neutrino oscillation, aiming its description in a progenitor star supernova environment. We begin calculating the wave packet size in many situations: solar and supernova plasma medium, considering nuclear interactions, as well as accelerators and reactors. This quantity is important to calculate the coherence length, which we compare with the oscillation length to verify if neutrino oscillation will or will not occur. Finally, we compare the wave packet treatment of neutrino oscillation with the plane wave formalism in a progenitor star supernova environment.
\end{abstract}

Keywords: Neutrino oscillation; Wave packets; Supernova

\section{INTRODUCTION}

Nowadays one knows that neutrinos really oscillate, based on the results of $\mathrm{SNO}$ [1] and KamLAND[2] which have solved the so-called solar neutrino problem[3] and estabilished the mass squared difference and mixing angle for the conversion of the first and second neutrino families. These results were very important, because one can reach a simple conclusion: neutrinos have mass and leptons are mixed, evidences that were also confirmed by K2K [4] and SuperKamiokande [5] experiments of atmospheric neutrinos.

Neutrino oscillations have been proposed in the late 50's by Pontecorvo[6-8]. The oscillations are generated by the interference of different massive neutrinos, which are produced and detected coherently because of their very small mass difference.

The theory of neutrino oscillations in the plane-wave approximation was developed in the middle 70's by Eliezer and Swift[9], Fritzsch and Minkowski[10], Bilenky and Pontecorvo[11], and reviewed by Bilenky and Pontecorvo in Ref.[12].

In 1976, Nussinov[13], for the first time, considered the wave packet nature of propagating neutrinos and inferred the existence of coherence length, beyond which the interference of different massive neutrinos is not observed. This is due to the different group velocities of different massive neutrinos that causes a separation of their wave packets. In 1996, Nussinov, Kiers and Weiss[14] first pointed out the importance of the detection process for the coherence of neutrino oscillations and discussed some implications of the wave packet approach.

In 1981, Kayser[15] presented the first detailed discussion of the quantum mechanical problems of neutrino oscillations, pointing out the necessity of a wave packet treatment.

There are two ways of facing the wave packet treatment of neutrino oscillation: using quantum mechanics[16-20] or using quantum field theory[21-27]. In this article we use the quantum mechanical approach, focusing in a supernova environment.

This article is organized as follow: in section 2 we describe a method for calculating the wave packet size in plasma medium; section 3 briefly reviews the wave packet formalism; section 4 presents our main results applied to the supernova case and section 5 concludes our work.

\section{WAVE PACKET SIZE}

The wave packet size is an important parameter and we have calculated it based on the model of reference [28]. The size is determined by the region where the neutrino is produced. Particularly, for a plasma medium, coherent emission of electronic neutrinos is constantly interrupted by the electromagnetic interaction between the particles that produce neutrinos and the plasma particles. Since $l$ is the mean free path of the particle which produces the neutrino and $v$ is its velocity, the wave packet size $\left(\sigma_{x}\right)$ can be determined by:

$$
\sigma_{x} \approx \frac{l}{v}
$$

By definition, the mean free path can be written as

$$
l=\frac{1}{\sigma N},
$$

where $\sigma$ is the respective cross section to be considered and $N$ is the medium particle number density. Considering equipartition of energy with the Coulomb field, we can write:

$$
\frac{3}{2} T \approx \frac{Z_{1} Z_{2} e^{2}}{b}
$$

and

$$
\frac{1}{2} m v^{2} \approx \frac{3}{2} T
$$

$T$ is the plasma temperature and $b$ is the impact parameter. Using expressions (1), (2) and (3) we can express the neutrino wave packet size as

$$
\sigma_{x} \approx 1.26 \times 10^{23} \frac{\sqrt{m} T^{\frac{3}{2}}}{Z_{1}^{2} Z_{2}^{2} N} \mathrm{~cm},
$$

where $T$ and $m$ are in $M e V$ and $N$ in $\mathrm{cm}^{-3}$. This expression can be used to calculate the wave packet size for a solar and a supernova media - see the second column of Table 5 and 6 , respectively. 
In the discussion above we have calculated the wave packet size based on electromagnetic interactions. Considering a supernova environment, in which matter density is very high, we consider the possibility that the wave packet size be determined by nuclear interactions, since neutrinos also can be produced by reactions such as $N N \rightarrow N N v_{i} \bar{v}_{i}$ (bremsstahlung), where $N$ represents a nucleus and $v_{i}$ a neutrino flavor. Besides, since core density is $\approx 10^{-14} \mathrm{~g} / \mathrm{cm}^{3}$, mean distance between particles will be $\approx 10^{-13} \mathrm{~cm}$, however we assume particles sometimes, by extrapolation, may reach Fermi distances and nuclear interactions will have a time scale shorter than electromagnetic interaction, changing the wave packet size. In a simple approach, if we consider the nuclear interaction a thousand times stronger than the electromagnetic interaction,

$$
V_{\text {strong }} \approx 1000 \times V_{\text {electro }},
$$

we will get wave packets $\approx 10^{-6}$ times smaller than wet got before in electromagnetic interactions.

TABLE I: Wave packet size considering nuclear interactions which can be important in a supernova environment. (Solar solution $\Delta m^{2} \approx 7.3 \times 10^{-5} \mathrm{eV}^{2}$ )

\begin{tabular}{|c||c||c||c|}
\hline Energy & $\sigma_{x}(\mathrm{~cm})$ & $L_{c o h}(\mathrm{~cm})$ & $L_{\text {osc }}(\mathrm{cm})$ \\
\hline $100 \mathrm{MeV}($ core $)$ & $10^{-20}$ & 7.75 & $3.40 \times 10^{8}$ \\
$10 \mathrm{MeV}($ core $)$ & $10^{-18}$ & 7.75 & $3.40 \times 10^{7}$ \\
$10 \mathrm{MeV}($ vsphere $)$ & $10^{-16}$ & 775.00 & $3.40 \times 10^{7}$ \\
\hline
\end{tabular}

TABLE II: Wave packet size considering nuclear interactions which can be important in a supernova environment. (Atmospheric solution $-\Delta m^{2} \approx 1.0 \times 10^{-3} \mathrm{eV}^{2}$ )

\begin{tabular}{|c||c||c||c|}
\hline Energy & $\sigma_{x}(\mathrm{~cm})$ & $L_{c o h}(\mathrm{~cm})$ & $L_{\text {osc }}(\mathrm{cm})$ \\
\hline $100 \mathrm{MeV}($ core $)$ & $10^{-20}$ & 0.56 & $2.48 \times 10^{7}$ \\
$10 \mathrm{MeV}($ core $)$ & $10^{-18}$ & 0.56 & $2.48 \times 10^{6}$ \\
$10 \mathrm{MeV}($ vsphere $)$ & $10^{-16}$ & 56.56 & $2.48 \times 10^{6}$ \\
\hline
\end{tabular}

So, for neutrinos produced in the supernova core, with $10 \mathrm{MeV}$ and $100 \mathrm{MeV}$ of energy, we got $\sigma_{x} \approx 10^{-20} \mathrm{~cm}$ and $\sigma_{x} \approx 10^{-18} \mathrm{~cm}$, respectivelly. For neutrinosphere, the wave packet size, considering nuclear interactions, will be $\sigma_{x} \approx$ $10^{-16} \mathrm{~cm}$, considering neutrinos with $10 \mathrm{MeV}$ mean energy. We resume these wave packet sizes values, as well as $L_{c o h}$ and $L_{o s c}$, in Table 1 , where we have used the mass squared difference from solar solution $\left(\Delta m^{2} \approx 7.3 \times 10^{-5} \mathrm{eV}^{2}\right)-$ the same is shown in Table 2 , but for atmospheric parameters $\left(\Delta m^{2} \approx 1.0 \times 10^{-3} \mathrm{eV}^{2}\right)$, and Tables 3 and 4 for two typical effective mass squared difference inside a progenitor star supernova $\left(\Delta \mu^{2} \approx 1.0 \times 10^{8} \mathrm{eV}^{2}\right.$ and $\Delta \mu^{2} \approx 1.0 \times 10^{4} \mathrm{eV}^{2}$, respectively).

Wave packets sizes are very important, since they are the main difference between wave packet formalism from the wave plane formalism in neutrino oscillation, as we will see in the following sections.

TABLE III: Wave packet size considering nuclear interactions which can be important in a supernova environment. (Effective mass squared difference $-\Delta \mu^{2} \approx 1.0 \times 10^{8} \mathrm{eV}^{2}$ )

\begin{tabular}{|c||c||c||c|}
\hline Energy & $\sigma_{x}(\mathrm{~cm})$ & $L_{\text {coh }}(\mathrm{cm})$ & $L_{\text {osc }}(\mathrm{cm})$ \\
\hline $100 \mathrm{MeV}($ core $)$ & $10^{-20}$ & $5.66 \times 10^{-12}$ & 0.00025 \\
$10 \mathrm{MeV}($ core $)$ & $10^{-18}$ & $5.66 \times 10^{-12}$ & 0.000025 \\
$10 \mathrm{MeV}($ vsphere $)$ & $10^{-16}$ & $5.66 \times 10^{-10}$ & 0.000025 \\
\hline
\end{tabular}

TABLE IV: Wave packet size considering nuclear interactions which can be important in a supernova environment. (Effective mass squared difference $-\Delta \mu^{2} \approx 1.0 \times 10^{4} \mathrm{eV}^{2}$ )

\begin{tabular}{|c||c||c||c|}
\hline Energy & $\sigma_{x}(\mathrm{~cm})$ & $L_{\text {coh }}(\mathrm{cm})$ & $L_{\text {osc }}(\mathrm{cm})$ \\
\hline $100 \mathrm{MeV}($ core $)$ & $10^{-20}$ & $5.66 \times 10^{-8}$ & 2.47 \\
$10 \mathrm{MeV}($ core $)$ & $10^{-18}$ & $5.66 \times 10^{-8}$ & 0.247 \\
$10 \mathrm{MeV}($ vsphere $)$ & $10^{-16}$ & $5.66 \times 10^{-6}$ & 0.247 \\
\hline
\end{tabular}

For explicity calculation of the wave packet size for reactor and accelerator neutrinos, see reference [28]. We present in Table 5 the wave packets sizes for these two situations.

\section{FORMALISM}

In this section we present the formalism of the wave packet oscillation first developing in vaccum and then generalized to the propagating in matter. In vacuum, we follow the wave packet formalism desenvolved by references [16] and [28]. We treat the problem unidimensionaly assuming gaussian wave packets with width $\sigma_{p}$ in momentum space, centered around average momentum $\left\langle p_{a}\right\rangle$ of a mass eigenstate.

The conversion probability, at a distance $X$ from the neutrino source, considering two families, is given by:

$$
\begin{aligned}
P_{\alpha \rightarrow \beta}(X)= & {\left[\sum_{a^{\prime}} \frac{\left|U_{\alpha a^{\prime}}\right|^{2}}{\left|v_{a^{\prime}}\right|}\right]^{-1} \sum_{a, b} U_{\beta a} U_{\alpha a}^{*} U_{\beta b}^{*} U_{\alpha b} \times } \\
& \exp \left\{i\left[\left(\left\langle p_{a}\right\rangle-\left\langle p_{b}\right\rangle\right)-\left(\left\langle E_{a}\right\rangle-\left\langle E_{b}\right\rangle\right)\left(\frac{v_{a}+v_{b}}{v_{a}^{2}+v_{b}^{2}}\right)\right] X\right\} \times \\
& \sqrt{\frac{2}{v_{a}^{2}+v_{b}^{2}}} \exp \left\{-\frac{X^{2}}{4 \sigma_{x}^{2}} \frac{\left(v_{a}-v_{b}\right)^{2}}{v_{a}^{2}+v_{b}^{2}}-\frac{\left(\left\langle E_{a}\right\rangle-\left\langle E_{b}\right\rangle\right)^{2}}{4 \sigma_{p}^{2}\left(v_{a}^{2}+v_{b}^{2}\right)}\right\} .
\end{aligned}
$$


TABLE V: Vacuum wave packet treatment for the solar, reactor and accelerator case.

\begin{tabular}{|c|c|c|c|c|c|}
\hline Experiment & $\Delta m^{2}\left(\mathrm{eV}^{2}\right)$ & $E(\mathrm{MeV})$ & $\sigma_{x}(\mathrm{~cm})$ & $L_{c o h}(\mathrm{~km})$ & $L_{o s c}(\mathrm{~km})$ \\
\hline Solar & $7.3 \times 10^{-5}$ & 10 & $3.15 \times 10^{-7}$ & $2.44 \times 10^{7}$ & $3.40 \times 10^{2}$ \\
Reactor & $7.3 \times 10^{-5}$ & 1 & $5.1 \times 10^{-4}$ & $3.95 \times 10^{8}$ & $3.40 \times 10^{1}$ \\
Accelerator & $1.0 \times 10^{-3}$ & $10^{3}$ & $1.0 \times 10^{2}$ & $5.65 \times 10^{18}$ & $2.48 \times 10^{3}$ \\
\hline
\end{tabular}

In the expression above, we see the first exponential term which is called phase factor and describes neutrino oscillation as a distance function. From this term we derive the oscillation length given by

$$
L_{o s c}=\frac{4 \pi E}{\Delta m^{2}}
$$

where $E$ is the neutrino energy and $\Delta m^{2}$ is the vacuum square mass difference $\left(\Delta m^{2}=m_{2}^{2}-m_{1}^{2}\right)$.

Second exponential term of expression (7) gives us the called damping and the exponential factors. From the damping term, we can derive the coherence length

$$
L_{c o h}=\frac{2 \sqrt{2} \sigma_{x} 2 E^{2}}{\Delta m^{2}}
$$

From equations (8) and (9) we write

$$
L_{c o h}>L_{o s c} \text {. }
$$

This condition essentially garantees that neutrino oscillation will occur. If neutrinos travel a distance $X, L_{o s c}$, given by (8), should be shorter than $X$ for one sees the oscillation pattern. But it is also fundamental that two mass eigenstates have to quantum interfere, in order to maintain coherence during the travel to present oscillation.

For neutrinos in matter, we follow the reference [29]. The development, in the adiabatic case, is very similar to the vacuum case. Conversion probability is given by

$$
\begin{aligned}
& P_{\alpha \rightarrow \beta}(X)=\sum_{a, b} U_{\alpha a}^{*} U_{\beta a} U_{\alpha b} U_{\beta b}^{*} \times \\
& \exp \left[-2 \pi i \frac{X}{L_{o s c}(X)}-\left(\frac{X}{L_{c o h}(X)}\right)^{2}-\frac{\left(E_{a}-E_{b}\right)^{2}}{8 \sigma_{p}^{2}}\right]
\end{aligned}
$$

with

$$
L_{o s c}(X) \equiv \frac{4 \pi E X}{\int_{0}^{L} d x \Delta \mu_{a b}^{2}(X)}
$$

and

$$
L_{c o h}(X) \approx \frac{4 \sqrt{2} \sigma_{x} E^{2} X}{\int_{0}^{L} d x \Delta \mu_{a b}^{2}(X)}
$$

The interpretation of $L_{o s c}$ and $L_{c o h}$, in (12) and (13) above, is similar to the vacuum situation and $\Delta \mu_{a b}^{2}$ is the mass squared diference in matter.

\section{RESULTS}

We divide the present section in two subsections: first, oscillation in vacuum; second, one discusses oscillation in matter; both are based on the wave packet formalism presented in Section 3.

\section{A. Vacuum situation}

Testing the solar and atmospheric solutions for solar neutri$\operatorname{nos}(E=10 \mathrm{MeV})$, for reactor neutrinos $(E=1 \mathrm{MeV})$ and for accelerator neutrinos $(1 \mathrm{GeV})$, we notice that the coherence length is much larger than the oscillation length, so neutrino oscillation will occur. Wave packet treatment is not necessary, since plane wave formalism of neutrino oscillation gives excellent results in analysing the oscillation data available and because condition (10) is fairly well satisfied in those environments. Table 5 shows some results of wave packet treatment for solar, reactor and accelerator situation. Note that even if we consider other values of allowed neutrino energies in those environments, as well as values of $\sigma_{x}$ varying of one or two orders from the values used in Table 5 , our conclusions will not change.

Something different happens when neutrinos are produced in a supernova environment, as can be seen in Table 6, which consider only electromagnetic interactions. Neutrinos from the core $(E=100 \mathrm{MeV}$, maximum energy of the spectrum), have oscillation length close to coherence length. Hence, we can not conclude that neutrinos will oscillate. In the last line of Table 6, we have used a typical value of effective squared mass difference: $L_{c o h}$ is still close to $L_{o s c}$. This is an indicative that we must investigate the situation inside the supernova environment. Neutrinos from neutrinosphere have a situation less critical. However, when we get an effective mass difference, oscillation length and coherence length are close to each other. It is important to notice that uncertainties in determination of the wave packet size could modify our results. As seen in Tables 1, 2, 3 and 4, where our calculations were made considering nuclear interations, condition (10) is never satisfied. 
TABLE VI: Vacuum wave packet treatment for the Supernova case.

\begin{tabular}{|c|c|c|c|c|c|}
\hline Experiment & $\sigma_{x}(\mathrm{~cm})$ & $E(\mathrm{MeV})$ & $\Delta m^{2}\left(\mathrm{eV}^{2}\right)$ & $L_{\text {coh }}(\mathrm{cm})$ & $L_{\text {osc }}(\mathrm{cm})$ \\
\hline \multirow{3}{*}{ Core } & $1.47 \times 10^{-12}$ & 100 & $7.3 \times 10^{-5}$ & $1.15 \times 10^{9}$ & $3.40 \times 10^{8}$ \\
\cline { 3 - 6 } & & 100 & $1.0 \times 10^{-3}$ & $8.43 \times 10^{7}$ & $2.50 \times 10^{7}$ \\
\cline { 3 - 6 } & & 100 & $1.0 \times 10^{8}$ & $8.43 \times 10^{-4}$ & $2.48 \times 10^{-4}$ \\
\hline \multirow{3}{*}{ - sphere } & $1.67 \times 10^{-10}$ & 10 & $7.3 \times 10^{-5}$ & $1.30 \times 10^{9}$ & $3.40 \times 10^{7}$ \\
\cline { 3 - 6 } & & 10 & $1.0 \times 10^{-3}$ & $9.47 \times 10^{7}$ & $2.50 \times 10^{6}$ \\
\cline { 3 - 6 } & & 10 & $1.0 \times 10^{4}$ & 9.47 & 0.25 \\
\hline
\end{tabular}

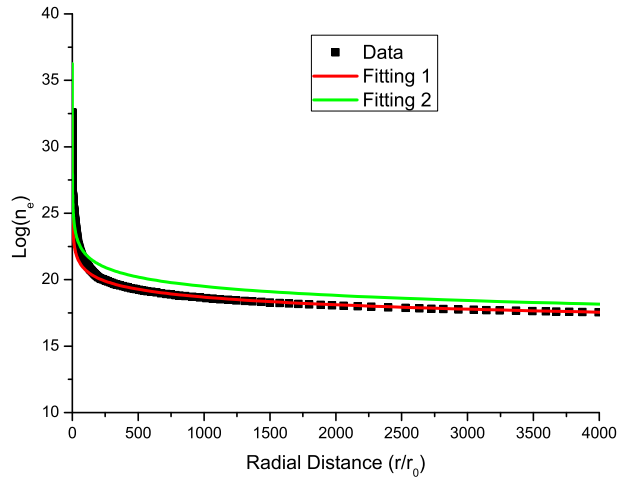

FIG. 1: Fitting of electronic density.

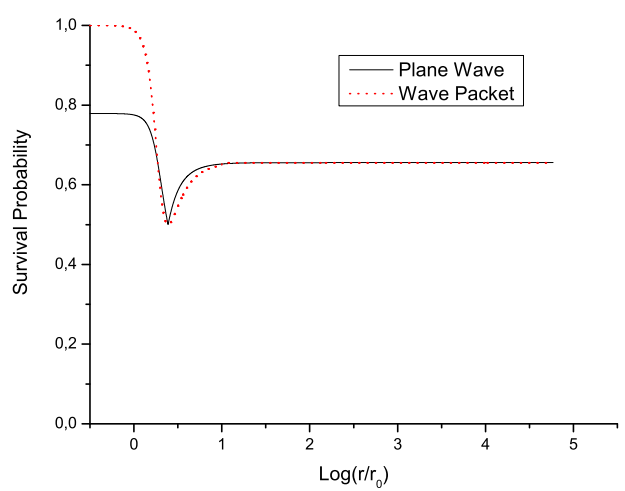

FIG. 2: Survival probability in plane waves (solid) and wave packet (dashed) $-40 \mathrm{MeV}$ and solar solution $\left(\Delta \mathrm{m}^{2} \approx 7.3 \times 10^{-5} \mathrm{eV}^{2}\right.$ and $\sin 2 \theta \approx 0.83) ; r_{0}=10^{4} \mathrm{~km}$.

\section{B. Matter situation}

The analysis inside the supernova claims the electronic density profile. The supernova data, with 15 solar masses, was provided by reference [30]. With this pre-supernova profile, we have done an analytical approach, fitting the electronic density by $\log \left(n_{e}\right)=25.50 \times\left(\frac{r}{r_{0}}\right)^{-0.045}\left(r_{0}=10^{4} \mathrm{~km}\right)-$ red fitting curve in Fig. 1 (best Fitting - fitting 1).

With this analytical expression of electronic density, neu-

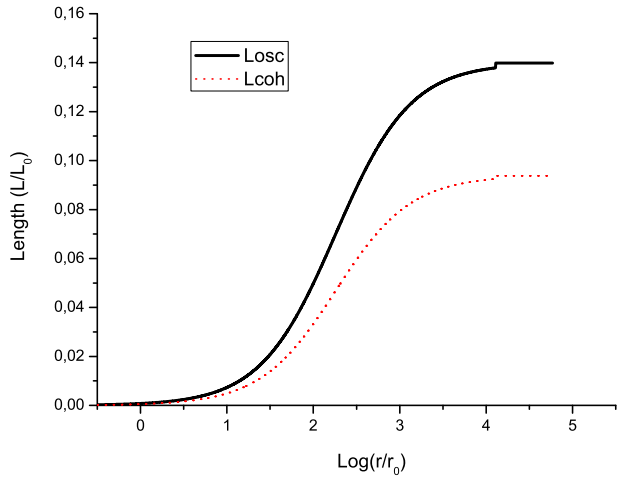

FIG. 3: $L_{o s c}$ (solid) and $L_{c o h}$ (dashed) $-40 \mathrm{MeV}$ and solar solution $\left(\Delta \mathrm{m}^{2} \approx 7.3 \times 10^{-5} \mathrm{eV}^{2}\right.$ and $\left.\sin 2 \theta \approx 0.83\right) ; r_{0}=10^{4} \mathrm{~km}$ and $L_{0}=10^{4} \mathrm{~km}$.

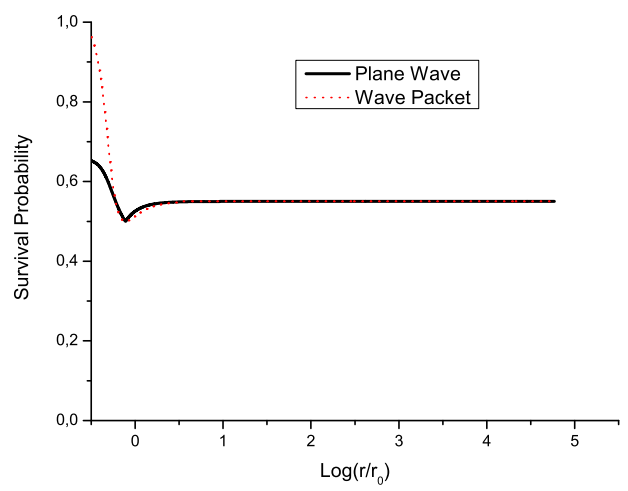

FIG. 4: Survival probability in plane waves (solid) and wave packet (dashed) $-40 \mathrm{MeV}$ and atmospheric solution $\left(\Delta \mathrm{m}^{2} \approx 1.0 \times 10^{-3} \mathrm{eV}^{2}\right.$ and $\sin 2 \theta \approx 0.95) ; r_{0}=10^{4} \mathrm{~km}$.

trino propagation will be adiabatic, since $\gamma \gg 1$, with $\gamma$ (adiabatic parameter) given by

$$
\gamma(x) \equiv \frac{(\Delta / E)^{2}}{2 \sqrt{2} G_{F}} \times \frac{(\sin 2 \theta)^{2}}{(\sin 2 \tilde{\theta})^{3}} \times \frac{1}{\left|\frac{d n_{e}}{d x}\right|}
$$

where $\Delta$ is the square mass difference in vacuum, $E$ is the 


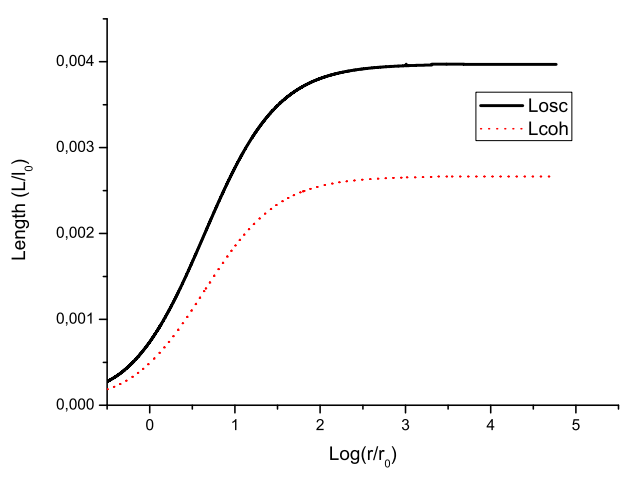

FIG. 5: $L_{o s c}$ (solid) and $L_{c o h}$ (dashed) $-40 \mathrm{MeV}$ and solar atmospheric solution $\left(\Delta m^{2} \approx 1.0 \times 10^{-3} \mathrm{eV}^{2}\right.$ and $\left.\sin 2 \theta \approx 0.95\right) ; r_{0}=$ $10^{4} \mathrm{~km}$ and $L_{0}=10^{4} \mathrm{~km}$.

neutrino energy, $G_{F}$ is the Fermi coupling constant and $\tilde{\theta}$ is the mixing angle in matter, which may be calculated by

$$
\sin ^{2} 2 \tilde{\theta}=\frac{\Delta^{2} \sin ^{2} 2 \theta}{(\Delta \cos 2 \theta-A)^{2}+\Delta^{2} \sin ^{2} 2 \theta},
$$

with $A=2 \sqrt{2} G_{F} n_{e} E$.

It is also important to calculate the effective mass square diference in matter $\left(\Delta \mu_{a b}^{2}\right)$ :

$$
\Delta \mu_{a b}^{2}=\sqrt{\Delta \cos 2 \theta-A)^{2}+\Delta^{2} \sin ^{2} 2 \theta} .
$$

Hence, with all parameters given above in hand, we calculate oscillation and coherence lengths, using expressions (12) and (13), and probabilities (11). With these values we can compare with results from plane wave formalism. Let us first consider wave packets sizes calculated by nuclear interactions (Table 1 ), with $\sigma_{x} \approx 10^{-20} \mathrm{~cm}$. Fig. 2 shows the comparison between survival probabilities in wave packet (dashed line) and plane waves (solid line) formalisms for oscillation parameters necessary for solar solutions $\left(\Delta \mathrm{m}^{2} \approx 7.3 \times 10^{-5} \mathrm{eV}^{2}\right.$ and $\sin 2 \theta \approx 0.83$ ) and neutrinos with $40 \mathrm{MeV}$ energy. We notice from Fig. 3 that, as long as neutrino propagates in the supernova environment, both probabilities become the same and freeze, because of the lost of coherence (oscillation length larger than coherence length - Fig. 3, where $L_{0}$ is $10^{4} \mathrm{~km}$ ). Figs. 4 and 5 show, also for neutrinos with energy $E=40 \mathrm{MeV}$, a similar situation and pattern, however for atmospheric solution $\left(\Delta m^{2} \approx 1.0 \times 10^{-3} \mathrm{eV}^{2}\right.$ and $\left.\sin 2 \theta \approx 0.95\right)$.
Neutrinos with $5 \mathrm{MeV}$ energy will respect the same situation and graphics will have the same pattern. For a solar and atmospheric solutions the wave packet treatment do not give any different result from the wave plane solution as we have just showed. LSND solution $\left(\Delta \mathrm{m}^{2} \approx 0.5 \mathrm{eV}^{2}\right.$ and $\left.\sin 2 \theta \approx 0.07\right)$ also does not introduce differences between both approachs. Also, if we modify the wave packet size to $10^{-18} \mathrm{~cm}, 10^{-16} \mathrm{~cm}$ or values calculated by electromagnetic interactions, as in Table 6 , no change will happen.

So, if we consider nuclear or electromagnetic interactions in the production of neutrino wave packets, one will have freezing on oscillation and coincidence between wave packet and plane wave probabilities curves.

\section{CONCLUSIONS}

We have discussed in this paper neutrino oscillation in the wave packet treatment, which we have applied to solar, reactor, accelerator and supernova neutrinos. This formalism introduces the damping term that has great importance since it elucidates the coherence between mass eigenstates. As we already know, solar, reactor, accelerator and high energy neutrinos have coherence length much larger than oscillation length, hence the wave plane formalism is enough for treating those cases. How about supernova neutrinos? Our proposal was to verify if the wave packet treatment of neutrino oscillation would give any different result from the plane wave formalism, using wave packets sizes calculated by electromagnetic and nuclear interactions. In Tables 1, 2, 3 and 4, where we have considered nuclear interactions, the coherence length is much smaller than the oscillation length, so one investigates, based on reference [29], the wave packet formalism influenced by matter. For a supernova with 15 solar masses, we verify that, for solar, atmospheric and LSND solutions, the conversion probability in the wave packet approach is the same as in the plane wave formalism. The same happens when we use wave packets sizes formed by electromagnetic interactions. We also notice the loss of coherence, since the oscillation length is higher than the coherence length, hence there will be a freezing in the oscillation pattern. So the wave packet formalism does not introduce any significant difference in neutrino oscillation physics.

\section{Acknowledgements}

F.R.T would like to thank CAPES and CNPq for financial support and Prof. Stan Woosley for providing supernova data.
[1] Q. R. Ahmad et. al., SNO collaboration, Phys. Rev. Lett. 89 , 011301 (2002).

[2] K. Eguchi et. al., KamLAND collaboration, Phys. Rev. Lett. 90, 021802 (2003).

[3] J. N. Bahcall, Neutrino astrophysics, Cambridge U. Press,
Cambridge, England, 1989.

[4] M. H. Ahn et. al., K2K collaboration, Phys. Rev. D 74, 072003 (2006).

[5] Y. Aschie et. al, Super-Kamiokande collaboration, Phys. Rev. Lett. 93, 101801 (2004). 
[6] B. Pontecorvo, Sov. Phys. JETP, 6, 429 (1957), [Zh. Eksp. Teor. Fiz. 33, 549 (1957)].

[7] B. Pontecorvo, Zh. Eksp. Teor. Fiz. 34, 247 (1958), [Sov. Phys. JETP 7, 172 (1958)].

[8] V. N. Gribov and B. Pontecorvo, Phys. Lett. B 28, 493 (1969).

[9] S. Eliezer and A. R. Swift, Nucl. Phys. B 105, 45 (1976).

[10] H. Fritzsch and P. Minkowski, Phys. Lett. B 62, 72 (1976).

[11] S. M. Bilenky and B. Pontecorvo, Nuovo Cim. Lett. 17, 569 (1976).

[12] S. M. Bilenky and B. Pontecorvo, Phys. Rept. 41, 225 (1978).

[13] S. Nussinov, Phys. Lett. B 63, 201 (1976).

[14] K. Kiers, S. Nussivov, and N. Weiss, Phys. Rev. D 57, 3091 (1998), hep-ph/9710289.

[15] B. Kayser, Phys. Rev. D 24, 110 (1981).

[16] C. Giunti, C. W. Kim, and U. W. Lee, Phys. Rev. D 44, 3635 (1991).

[17] C. Giunti, C. W. Kim, and U. W. Lee, Phys. Lett. B 274, 87 (1992).

[18] A. D. Dolgov, A. Y. Morozov, L. B. Okun, and M. G. Shchepkin, Nucl. Phys. B 502, 3 (1997), hep-ph/9703241.

[19] C. Giunti and C. W. Kim, Phys. Rev. D 58, 017301 (1998), hep- $\mathrm{ph} / 9711363$.

[20] C. Giunti, Physica Scripta, 67, 29 (2003), hep-ph/0202063.

[21] M. Beuthe, Phys. Rev. D 66, 013003 (2002), hep-ph/0202068.

[22] M. Beuthe, Phys. Rept. 375, 105 (2003), hep-ph/0109119.

[23] C. Y. Cardall, Phys. Rev. D 61, 073006 (2000), hep$\mathrm{ph} / 9909332$.

[24] C. Giunti, JHEP, 11, 017 (2002), hep-ph/0205014.

[25] C. Giunti, C. W. Kim, J. A. Lee, and U. W. Lee, Phys. Rev. D 48, 4310 (1993), hep-ph/9305276.

[26] C. Giunti, C. W. Kim, and U. W. Lee, Phys. Lett. B 421, 237 (1998), hep-ph/9709494.

[27] K. Kiers and N. Weiss, Phys. Rev. D 53, 537 (1996), hep$\mathrm{ph} / 9506271$.

[28] C. W. Kim and A. Pevsner, Neutrinos in Physics and Astrophysics, Harwood Academic Press, Chur, Switzerland, 1993, Contemporary Concepts in Physics, Vol. 8.

[29] J. T. Peltoniemi and V.Sipiläinen, JHEP 0006, 011 (2000), hep$\mathrm{ph} / 0004162$.

[30] Supernova data with 15 solar masses (www.supersci.org) 